\title{
The Cluster Between Internet of Things and Social Networks: Review and Research Challenges
}

\author{
Antonio M. Ortiz, Member, IEEE, Dina Hussein, Soochang Park, Member, IEEE, Son N. Han, Student Member, \\ IEEE, and Noel Crespi, Senior Member, IEEE
}

\begin{abstract}
The cluster between Internet of Things (IoT) and Social Networks ( $\mathrm{SN})$ enables the connection of people to the ubiquitous computing universe. In this framework, the information coming from the environment is provided by the IoT, and the SN brings the glue to allow human-to-device interactions. This article explores the novel paradigm for ubiquitous computing beyond IoT, denoted by Social Internet of Things (SIoT). Although there have been early-stage studies in social-driven IoT, they merely use one or some properties of SIoT to improve a number of specific performance variables. Therefore, this article first addresses a complete view on SIoT and key perspectives to envision the real ubiquitous computing. Thereafter, a literature review is presented along with the evolutionary history of IoT research from Intranet of Things to SIoT. Finally, this article proposes a generic SIoT architecture, and presents a discussion about enabling technologies, research challenges, and open issues.
\end{abstract}

Index Terms-Internet of Things, Social Internet of Things, Social Networks.

\section{INTRODUCTION}

We are standing on the brink of a new era with real ubiquitous computing and communication where many gadgets, such as sensors, RFID tags, and smart electronic/electromechanical devices, surrounding us will be on the network [1], [2]. The gadgets would disappear and weave themselves into the fabric of our everyday life to work in concert to support us in carrying out daily life activities, tasks and rituals in an easy, natural way using information and intelligence, hidden in the network connecting the gadgets. This pervasive paradigm known as Internet of Things (IoT) might increase the value of information generated by the number of interconnection between people and gadgets, denoted by things, and transformation of the processed information into knowledge for the benefit of mankind and society [3].

IoT will usher in a wide range of smart services and applications to cope with many of the challenges that individuals and organizations face in their everyday lives via allowing humans and things to be connected with either anyone or anything, in any place, at any time [1][4].

The IoT vision of pervasively connecting billions of things is able to interact with the environment around us and receive information on its status that was previously not available by simply looking at a set of things [5]. In other words,

A. M. Ortiz, D. Hussein (Corresponding Author), S. Park, S. N. Han and N. Crespi are with the Department of Wireless Networks and Multimedia Services, Institut Mines-Telecom, Telecom SudParis, Evry-Cedex, 91011 France (email: \{antonio.ortiz_torres, dina.hussein, soochang.park, son.han, noel.crespi\}@it-sudparis.eu).

Manuscript received October 31, 2013 while previous Intranet of Things [5][6], which is a local network of a set of things such as Wireless Sensor Networks (WSNs), Machine-to-Machine (M2M), and smart homes, can only extract regional information containing specific content from the things, IoT can provide large scale, comprehensive, and historical information by collaborating between different intranets of things even if they have heterogeneity regarding devices, local communication technologies, and deployment goals. Furthermore, IoT enables the creation and composition of new services and applications, offering to individual users a new ecosystem where different intranets of things can collaborate.

Humans usually interact with others in a wide variety of relationships during their everyday life. Also, they would utilize many smart services and applications from IoT to improve their life quality. Hence, quality of experience (QoE) of those services and applications depends on how to satisfy the needs driven from the relations among humans. In addition, a high degree of correctness of each need is practically derived by the collaboration with humans in the relationship related to a service. In IoT, as mentioned above, an individual user connects to the other(s) via legacy networks; on the other hand, sets of things collaborate with each other via the Internet for offering information to smart services and applications, while each user uses them. Thus the IoT follows two interaction paradigms: human-to-human and thing-to-thing, and then humans merely utilize data from things as an old-fashioned client-server interaction model [7][8]. It means that IoT so far does not adopt a true connection between humans and things, i.e., human-to-thing, for real ubiquitous computing [6].

In order to practically integrate the ubiquitous computing in our future daily life with high QoE, we need to improve the connectivity of all the relationships between users and things, and to enhance the availability of computational power via sets of things surrounding us. Therefore, we take into consideration Social Networks (SN) of all entities (i.e., humans and things) for ubiquitous computing as an evolution beyond the IoT. In other words, things should be socialized for allowing humans to establish relationships with them in an easy way. It does not only mean physical connections between humans and things, but also logical configurations of social communities involving humans as well as things. This logical configuration can be realized through exhibiting features from people's $\mathrm{SN}$ and adopts them for the suggested universal SN of all entities. The feature set can include the interactivity scheme, profiling system, recommendation, and mashup of services. This SN assists in the emerging of new communities driven 
by increasing sociality, inherited from traditional SNs, could give the chance to understand user requirements, and thus they also improve the availability based on trust in each community.

In fact, increasing the availability of processing power would be accompanied by decreasing the visibility [1][9]. Thus, this novel pervasive vision with improved sociality is denoted by Social Internet of Things (SIoT).

Consequently, new ecosystems coming out when clustering social networks and IoT allow the enrichment of/from both paradigms since IoT provides the connection to the physical world by sensing and actuating while social networks contribute to many of the daily aspects of the human world.

This article explores the evolutionary history of what has been called SIoT, analyzes the gaps in social-driven IoT studies in early stage, with an eye on future ubiquitous computing (i.e., SIoT), and comes up with a novel architecture design that integrates different components from previous proposals, and with newly driven requirements and research challenges for SIoT.

The rest of this article is organized as follows. In Section II, we address the role of SNs for IoT evolution as well as discussion of the SIoT values. Then, we go through the history of technical improvements from WSNs to IoT in Section III. Section IV provides literature review of socialdriven IoT studies based on architectures, current research trends, applications, and SIoT in the industry. In Section V, we discuss the new challenges and open issues of SIoT with the architectural elements that will pave the way toward this future-driven SIoT paradigm. Finally, Section VI gives some concluding remarks.

\section{Social Internet of Things}

In this section, we address three questions to help in understanding the concept of SIoT and its main contributions to the current technology. Next, we discuss each question trying to raise concerns and seek solutions to them.

\section{A. Why are we considering Social Network principles to be integrated with real ubiquitous computing?}

The term Community of Interest (COI) generally refers to a set of communicating entities [1][10]; it may also be further extended to refer to a set of communicating entities engaged in wanted communication, namely "good COI" [2]. Individuals in such COI share various resources in online and offline settings. They share social relationships, interests, and contextual and multimedia resources. Also, in some scenarios they share physical devices and objects, i.e., office/home appliances. While social relationships and contextual data shared within an online community are considered as productive resources that would facilitate cooperation for mutual benefits [3], SIoT suggests extending this principle to integrate physical elements and their contextual data from the real world into the social capital to reach a better understanding and modelling of the users real needs and objectives.

In ubiquitous computing environments, realizing a vision of Ambient Intelligence [11] where many different devices, called things, will gather and process information from many different sources to control both physical processes and interactions with users, we can find diverse interaction models among devices and humans: human-to-human, thing-to-thing, and human-to-thing. They aim at supporting a wide variety of services and applications for individuals and social communities. In fact, when various scenarios of thing-to-thing interaction provide local/global information to services, users trust and utilization of these services are considered as the core value in ubiquitous computing environments. Consequently, close interaction of human-to-thing form the next wave of SIoT. Therefore, it eventually achieves optimization of the best services to be offered to users with improved QoE. Brought by the notion of trust in social communities, this shift from thingto-thing pairing towards human-to-thing implies that users are no longer only consumers of services but they also participate in creating services. This transit brings up new challenges in terms of context and communications as well as benefits. In this article we mainly focus on two benefits: (i) improving the QoE and (ii) enhancing the collaboration within communities involving humans and things.

On the other hand, achieving collaboration is one of the major goals of SNs. With the wealth of social and contextual data shared and stored, online presence of individuals and communities is no longer passive. They collaborate by publishing data and content aiming to meet their needs in everyday life. In SIoT vision, users and mostly devices are the core contributors to create services as well as consumers of services. The wheel starts turning from individuals and communities, it gathers social data and communicates with devices shared on the physical world to eventually offer a set of services and an enhanced collaboration back to communities and individuals.

As the paradigm for Ubiquitous Computing [9] implies making the computational power available anytime/anywhere and exploits it toward the benefit of mankind and societies [4], this paradigm will not be completed without understanding society needs and challenges. Weaving the social/contextual data and relationships available within online social networks with physical thing worlds will ultimately reach this goal.

\section{B. Why SIoT is the next step?}

Future ubiquitous computing will usher in a wide range of smart services and applications to cope with many challenges that individuals and organizations face in their everyday lives via allowing humans and things to be connected with either anyone or anything, in any place, at any time. While IoT studies [1][12] have typically mentioned communication to physical world by sensing or actuating through many of different devices to be the biggest novelty, SIoT paradigm however raises important concerns about why and how to utilize these services and applications. For this objective, there are two considerations as shown in Fig. 1: 1) increasing sociality (or connectivity) and 2) improving pervasiveness (or availability).

In order to settle on all the properties of real ubiquitous computing in our future daily life with high QoE, we need to improve the connectivity of all the relationships between 


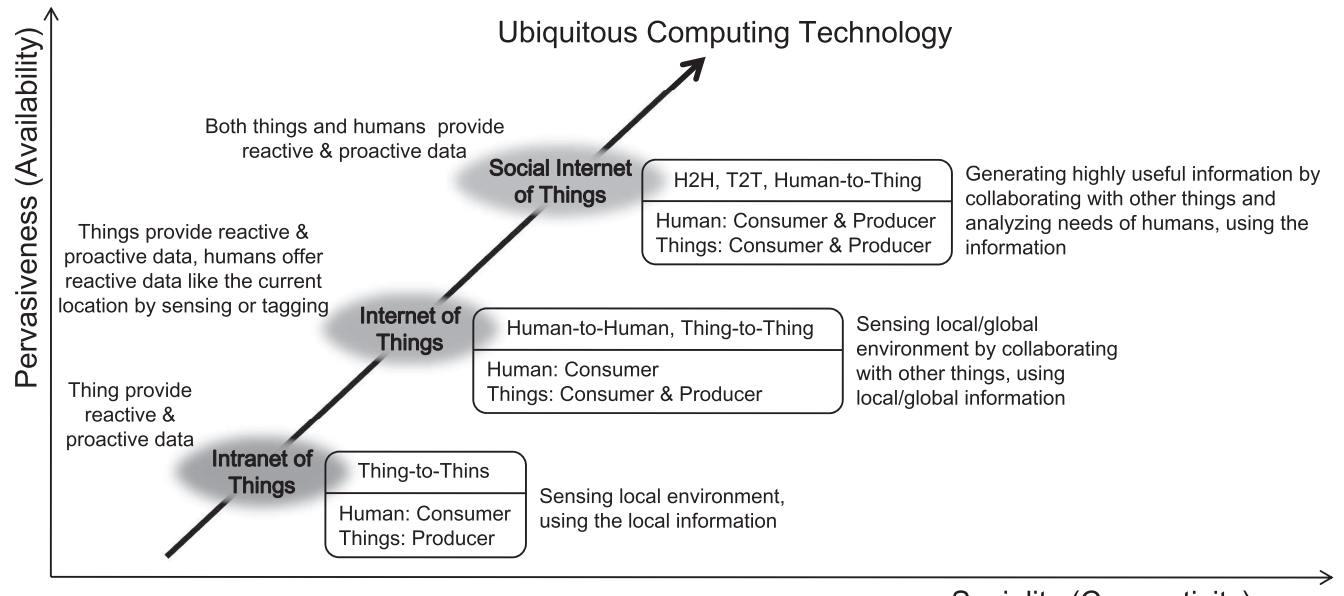

Sociality (Connectivity)

Fig. 1. Evolutionary History of Ubiquitous Computing Technology.

humans and things. As mentioned above, in human society a person typically functions both as a consumer and as a producer to communicate with the others; moreover, in either physical or online world, social networks could provide longterm (or proactive) and on-demand (or reactive) information, e.g., needs, interests, locations, demographic properties, relationship characteristics, etc., of individuals or communities through collaboration with each other. Storing, processing, and utilizing these social aspects could eventually help to improve QoE.

In pervasive environments, users tend to access services any time and anywhere using any kind of devices through any kind of communication networks. When overseen through the SIoT vision, pervasiveness will imply the weaving of actual, virtual, and physical things into SIoT.

In SIoT, users themselves can unintentionally participate in the process of improving QoE through things they use and share on a daily basis which represent their interests and needs. Moreover, things will collaborate with other diverse things to satisfy their objectives which are driven from the humans. In this sense, humans and things are no longer seen as separate nodes inside a network, and their objectives and needs will weave together forming what we call SIoT. That is, such close link formation between humans and things would cause increasing availability of both elements (i.e., humans and things) as well as assuring their transparency. The two previously mentioned notions of transparency and availability will eventually drive us to the highly pervasive world, as promoted by future driven ubiquitous computing systems.

\section{What are the key perspectives for future-driven SIoT?}

In order to achieve an actual implementation of the seamless integration between social and IoT worlds and to gain the benefits promoted by the SIoT vision discussed above, certain perspectives need to be considered. Figure 1 illustrates these perspectives together with the evolution history of ubiquitous computing technology.
- Interactivity Perspective: the pairing between humans and things in IoT can take place in two forms: human-tohuman or thing-to-thing interaction and it can be achieved using the normal physical interaction in case of humans or various computer networks in case of things. In the current state of the art, most of the contributions focus on a single form of communication at a time whereas in this article, we claim that implementing human-to-thing interactions is essential to achieve the completed vision of SIoT. Such kind of communication opens the door to another level of pervasiveness in IoT environments and in fact opens the door to many other networking and communication issues that must be tackled.

- Collaboration Perspective: this perspective appears to be the most important one in order to realize a complete convergence of both the social and IoT worlds because it endorses human-to-thing interactions. We explore the roles of humans and things. Considering social values, SIoT ultimately allows humans and things to act as producers or consumers, and this leads to increasing collaboration among all the entities as well as eventually enhancing QoE.

- Handled-data Perspective: it is also very important to consider the kind of data acquisition and handling techniques needed to be considered in pervasive environments. We categorize data acquisition techniques into two categories: (i) proactive data acquisition that is commonly uses crawling techniques, learning algorithms, or various data analysis algorithms, and (ii) reactive data acquisition which usually operates in a real time manner using various data mining and query techniques. In SIoT, both kinds of data acquisition can be used depending on the given scenarios. For instance, location information of a user could indicate either the current location for an ondemand query or the historical trajectory by learning and analyzing. Also, this property could cause the same situation for temperature monitoring by things. 


\section{FROM WSN TO SIOT}

Wireless Sensor Networks (WSNs) appeared by the end of the last century, gaining wide attention from the research community and from a number of companies that developed commercial products using this technology. WSNs, composed of resource-constrained (i.e., battery, processing, storage, etc.) devices opened a research topic, where many studies started to propose new protocols, architectures, developments, and applications with the aim of extracting all the potential of this new paradigm [13]. Many proposals were oriented to raise new ideas, while others were focused on the adaptation of existing developments to the requirements of this new technology.

WSNs have typically no infrastructure. They consist of a variable number of low-power nodes (from few tens to thousands) that can communicate with each other, covering a region and working together to monitor different variables of the environment that are measured by sensors included in the nodes. Commonly, there exists a base station, central node, gateway, or sink, that gathers the data coming from network nodes to enable further data analysis.

Applications for WSNs include environmental monitoring (e.g., flood detection, precision agriculture, forest fire detection and tracking, etc.), military target tracking and surveillance, health (e.g., telemonitoring of human physiological data, patient tracking, drug administration, etc.), home and building monitoring and automation, security and surveillance, vehicular applications, warehouse management, etc. [14]. Moreover, WSNs are an important part of other technologies such as Body Area Networks [15], Vehicular Networks [16], Home Automation and Domotic [17], and Smart Cities [18].

One of the main issues of WSNs is the availability of data (i.e., who and how data can be accessed). Generally, WSNs are proprietary deployments and data is private, and the use of no-standard communication approaches is very common. In that way, WSNs are isolated, and both users and devices cannot take advantage of other deployments, so increasing costs and reducing the functionality. From this point comes the idea of IoT, a network where anything, anywhere, anyone, at anytime is connected, providing communication among different networks through the use of Internet.

IoT has been defined as a world-wide network of interconnected objects uniquely addressable, based on standard communication protocols [19]. However, the main challenge of this technology is to find the appropriate approach to integrate generic objects (i.e., devices) into a common framework.

When integrating such as number of devices with the communication capabilities expected from IoT, the application scope automatically grows, including new applications such as aerospace and aviation, automotive, telecommunications, healthcare, independent living, pharmaceutical industry, retail, logistics and supply chain management, smart cities, manufacturing, advanced environment monitoring, agriculture and breeding, media and entertainment industry, insurance, recycling, etc. [20], [21].

As the interest of the community grows for the IoT paradigm, so does the need for sharing IoT data, services and applications, and the integration of ubiquitous computing in the everyday life also has to comply with social interactions. At this point is where social networking principles are progressively being integrated in the IoT, so bringing on the paradigm known as Social IoT.

The next section reviews the current technological developments that are oriented to include social skills in the loop, from the pioneer approaches that propose to publish sensor data in micro-blogging networks to the well-established architectures for SIoT, detailing the basics of this new paradigm, new application domains and industrial developments that are based on this technology.

\section{Current Trends: IoT is Becoming Social}

Although the notion underlying the integration of social aspects with IoT as promoted by SIoT is still new and in an early stage of investigation, some research contributions already paved the way by offering solutions for engaging people, through social networks, along with distributed sensors and embedded devices as a way to enhance services and applications. In [22], for instance, the IoT paradigm is proposed to be enriched with Twitter-communication capabilities to post updates and information about the status of some on-going tasks and activities. Similarly in [23], an approach based on WSN is presented making also use of Twitter to publish and share sensory data and resources. In [24], the IoT architecture is considered as a social organization framework to federate ubiquitous IoT architecture. Other approaches propose to extended the IoT through the use of social networks APIs, for instance, [25] suggests a platform that enables people to share their World Wide Web-enabled devices so that others can use them.

\section{A. SIoT Paradigm}

The SIoT paradigm represents an ecosystem that allows people and smart devices to interact within a social framework. On top of this framework, applications and services can be offered relying on Web technologies. To realize this framework some major building blocks should be provided. In this section we sum up these key aspects that constitute the basis of SIoT: the social role, intelligence, socialized devices, and everything as a service.

- Social Role: In [26], [27], [28] the social role initiates from users' social network, where the argument to bring it into IoT world is to guarantee the network navigability, and an efficient service discovery. Similarly in [29] the social role is promoted by the use of popular online social networks and their APIs to maintain a social structure and relationships with smart objects. Also, this suggested social structure allows to share smart objects relying on the trust provided by the community. In [30], users' social networks accounts can help in service operation for SIoT, for instance, to utilize geo-location data or publish devices' status and updates. The social role appears in [31] in terms of utilizing social networks as an interface to control smart objects.

- Intelligence: In [27] the concept of intelligence is mentioned as an essential component of the SIoT paradigm 
which is responsible for starting, updating and terminating the objects' relationships in SIoT. This is not only the scope of intelligence, in [29] the notion of intelligence is to allow dynamic thing-to-thing service discovery where smart objects can understand each others' services in an automated way. The work presented in [32] envisions that intelligence is implemented as a middleware combining many technologies such as ontologies, techniques for processing user generated content, and recommendation techniques. In short, intelligence in literature appears to be a sort of decision maker to drive the use of services.

- Socialized Devices: The concept of socialized devices as brought in by very early contributions in SIoT such as [26], [27], [28], [29] may be the most essential architectural component because it implies the mechanism which various smart objects and embedded devices will use to communicate with people through the Internet. [33] introduced the idea of collaboration between social networks and smart objects, an analysis of the features of social devices is also presented in this article, focusing on the vision of enabling smart devices to "talk" with other objects, to share experience about certain situations and to seek help. In [29], social devices rely on Web protocols to communicate with users through a social network environment.

- Everything as a Service: The notion of turning objects and SNs functionalities into services and enable them to be easily discovered and integrated with various other services has been presented in the literature to utilize the convergence between the social and devices roles as promoted by SIoT. Thus, people can share the services offered by smart objects with friends or objects [34]. This kind of sharing implies the use of the social role to discover and promote services. However, the concept of turning "everything" to a service is presented in [27] as a wider vision, by associating smart objects with the services they deliver. The discovery of new services to be utilized or mashed-up with other services can take place endorsed by the social role, where a user can discover trustworthy services within her/his social community.

TABLE I

SIOT ARCHITECTURE DESIGN

\begin{tabular}{lcccc}
\hline \hline Contribution & SR & Intll. & SD & EaaS \\
\hline Atzori et al. [26][27][35] & & & $\checkmark$ & $\checkmark$ \\
Atzori et al. [28] & $\checkmark$ & & & $\checkmark$ \\
Zhang et al. [31] & & $\checkmark$ & & \\
$\begin{array}{l}\text { Pintus } \text { et al. [30] } \\
\text { Ciortea } \text { et al. [36] } \\
\text { Guinard } \text { et al. [29] }\end{array}$ & $\checkmark$ & $\checkmark$ & & \\
\hline \hline \\
\hline Social Role (SR), Socialized Devices (SD), \\
Everything as a Service (EaaS)
\end{tabular}

\section{B. SIoT Architecture}

In the literature, the Web of things (WoT) is presented as an evolutionary step following the IoT paradigm [29] where in the former, smart objects and people relies on Web standards and protocols to interact as peers in an integrated ecosystem. The convergence between the social aspects in WoT and IoT paradigms formed the focus of many research papers; however, the two terms themselves are being alternatively used in the literature to refer to almost the same paradigm. In this article we stick with the term SIoT; however, we consider contributions in the field of SWoT as well. Table I summarizes the research papers with their mainly presented architectural components presented in Section IV-A.

TABLE II

SIOT RESEARCH TRENDS

\begin{tabular}{lccccc}
\hline \hline Research Contribution & SWS & SC & LBA & SNGA & TM \\
\hline Atzori et al. [27] & $\checkmark$ & & & & \\
Pintus et al. [30] & $\checkmark$ & & & & \\
Guinard et al. [34] & $\checkmark$ & & & $\checkmark$ & \\
Misra et al. [37] & & & & $\checkmark$ & \\
Blackstock et al. [38] & & & & & $\checkmark$ \\
Guinard et al. [29] & $\checkmark$ & & & & \\
Lequerica et al. [39] & & $\checkmark$ & $\checkmark$ & & \\
Makitalo et al. [40] & & $\checkmark$ & $\checkmark$ & & \\
AN Jian et al. [41] [42] & & $\checkmark$ & $\checkmark$ & & $\checkmark$ \\
Michelle Nitti et al. [43] & & & & & $\checkmark$ \\
Fenye Bao et al. [44] & & & & \\
\hline \hline
\end{tabular}

Semantic Web Service (SWS), Social Cognition (SC), Location Based Awareness (LBA), Social Net. Graph Analysis (SNGA), Trust Manag. (TM)

\section{SIoT Research Trends and Applications Domains}

The potentials offered by SIoT make possible the development of a huge number of applications, however due to the relatively novelty of the concept it has not been fully exploited in many applications. We split contributions into two categories: research trends utilizing the social aspects in an IoT environment to offer a higher quality of service, and some partially or fully implemented prototypes that propose the idea of integrating the social networks with IoT to improve aspects our daily life. Various research trends exist in the literature, although they are not originally presented under the SIoT umbrella, they can still fit into it by combining the social role with IoT. We sum up those in Table II. The research trends includes semantic web services environments to enable devices and social service discovery and mash-up. Other trends focus on realizing some social aspects from online social networks or peer-to-peer social networks to offer social aware services. Location based awareness is another research trend where location data are collected and processed to offer customized services. The analysis of the social network graph has been also considered to gain understanding of the social relationships. Finally, trust management is offered to orchestrate the process of service discovery. Table III shows some of prototype examples in the field of SIoT.

\section{Industrial Approaches for SIoT}

Many companies in different industrial sectors have already approached this new trend by using cutting-edge technologies to develop new products and services that will drive the next wave of innovation in SIoT. These social devices ranging from low-cost sensors to powerful embedded systems can gather 
TABLE IV

Social Internet of Things Commercial Products

\begin{tabular}{llllll}
\hline \hline Product & Manufacturer & Industry & Social Features & Commun. & Open APIs \\
\hline Social Vending System & PepsiCo & Beverages & $\begin{array}{l}\text { Social network of vending machine } \\
\text { Gift/share a beverage to a friend } \\
\text { with video messages }\end{array}$ & Wired & No \\
Nike+ FuelBand & Nike & Apparel, Accessories & $\begin{array}{l}\text { Fitness-tracking wristband social network. } \\
\text { FuelBand users share their fitness data }\end{array}$ & Wireless & Yes \\
Nuvant MCT & Corventis & Healthcare & $\begin{array}{l}\text { Noninvasive, ambulatory arrhythmia monitoring } \\
\text { Physicians, patients, and families coordinate }\end{array}$ & Wireless & No \\
Good Night Lamp & Good Night Lamp & Appliances & Wired an ambient way and availability & No & Wired \\
& & & & & \\
\hline \hline
\end{tabular}

TABLE III

SIOT PROTOTYPE DOMAINS

\begin{tabular}{lcccc}
\hline \hline $\begin{array}{l}\text { Research } \\
\text { Contribution }\end{array}$ & Gastronomy & $\begin{array}{c}\text { Smart } \\
\text { shopping }\end{array}$ & $\begin{array}{c}\text { Smart } \\
\text { City }\end{array}$ & $\begin{array}{c}\text { Smart } \\
\text { Home }\end{array}$ \\
\hline $\begin{array}{l}\text { Console } \text { et al. } \text { [45] } \\
\begin{array}{l}\text { Ceipidor } \text { et al. } \text { [46] } \\
\text { Stavroulaki } \text { et al. } \text { [47] }\end{array}\end{array}$ & $\checkmark$ & & & \\
Hussein et al. [48] & & $\checkmark$ & $\checkmark$ & \\
\hline \hline
\end{tabular}

data and communicate these data over the Internet to social networks of people and devices who can respond to a problem, deliver a service, or sell a solution [49]. Table IV summarizes a number of off-the-shelf commercial social devices. These are very appealing products that bring the new experience of SIoT to the customers. Though, except products from Nike+ that provide open APIs for third-party providers to interact with their devices, most of the products available on the market are still in isolated islands, which would be a challenge for the future interoperability of SIoT products.

\section{E. Standardization activities for SIoT}

In order to standardize the diverse aspects of SIoT, several standardization bodies accept contributions related to the consideration of Social Network properties into the IoT, and also the inclusion of IoT devices into the Social Networks structure. ITU- ${ }^{1}$ for instance, is focused on information and communication technologies, and proposals such as the one presented in [50], that combines Social Networks and IoT, are part of the new era on standardization activities; oneM2 $\mathrm{M}^{2}$, initiated in 2012, aims at creating a common M2M service layer to ensure that Machine-to-Machine communications can effectively operate in a worldwide manner. Considering the importance of Social Networks in the framework of IoT and thus in M2M, it is expected that a number of contributions to oneM2M are oriented to SIoT.

\section{Discussion - Challenges And Open Issues}

As stated in many research works, Social IoT appears to be the next step in the evolution of ubiquitous computing. However, there are still a number of challenges and open issues that should be faced by the research community in order to mature this technology.

\footnotetext{
${ }^{1}$ http://www.itu.int/

${ }^{2}$ http://www.onem2m.org/
}

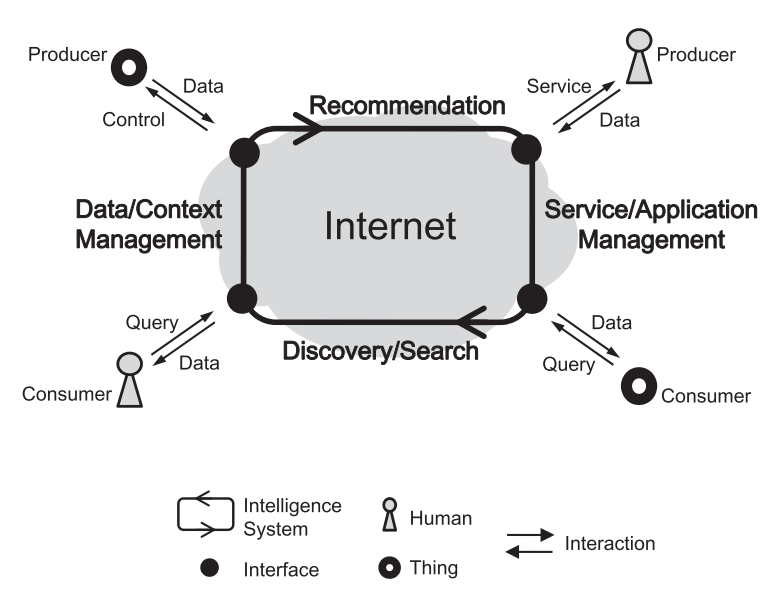

Fig. 2. Future SIoT Architecture.

After having illustrated the evolution from WSN to SIoT, described the main stages of this transformation, reviewed the literature and the commercial approaches associated to Social IoT, this section exposes the main research directions that will help to create this technology. First, we provide a general purpose architecture for SIoT that integrates the main architectural components proposed in the literature. Later we analyze technological developments, both hardware and software, that will make possible the successful operation of the paradigm. Finally, we focus on non-functional requirements, that constitute an essential part of the SIoT ecosystem.

\section{A. Architecture - General Design}

To summarize our vision towards a future driven SIoT, we consider the following elements to be part of the architecture: 1) actors (i.e., smart things and users), 2) an intelligent system to manage and orchestrate actors' interactions, 3) an interface for actors to enable interactions, 4) the Internet to provide open access among all the involved entities. Next we discuss about each element in the architecture in detail (see Fig. 2).

- Actors: the SIoT paradigm suggests a democratic environment, where both humans and things can participate equally by publishing data and recieving control commands for managing data being produced. These data can be represented as profiling data or simply responses to queries sent by users and/or devices. Queries in this sense can be sent to find the closest node, the most reliable node 
or service, or to simply receive updates about weather or a certain device status. In return, humans and things can receive services, or recommendations for services to consume, that fulfill current situations and long term objectives i.e, power efficiency plan for a smart grid in a smart home.

- Intelligent system: is responsible for managing and orchestrating the whole interactions undertaken by the actors, we actually sum up the main sub systems to be part of the intelligent system such as service and applications management, recommendation, service discovery and search, and data and context management.

- Interface: all the interactions with the system take place through an interface that enables the input of data and queries, as well as it provides the requested output (i.e., control commands or services).

- Finally, the Internet act as a communication medium to bring smart devices with their services to the users and also to allow them to interact with their devices and services.

\section{B. Enabling Technologies}

In order to have a functional platform that successfully implements the requirements and achieves the objectives of SIoT, some advances in the technological field must be reached.

With the objective of making any entity in the network (i.e., users and devices) identifiable and retrievable, it is necessary to map a unique identifier to each component [20]. Apart from the public names or nicks that will be used by the final users, the system needs an addressing scheme that enables different management tasks such as identity administration and authentication to ensure that the heterogeneity of individuals in the network can be identified.

Regarding the hardware developments, there is nowadays a vast variety of devices that can be adapted to be part of the SIoT. Sensor and actuator devices from WSN, M2M, domotic, etc., are good candidates to take part in this new paradigm. However, these devices usually cannot directly connect to the Internet, and need the use of gateways to transfer their readings and receive commands. Since many of the capabilities of SIoT are going to be accessed through web services, the use of web-enabled devices will facilitate the deployment and further use of this paradigm. Moreover, new deployments should address the energy-efficiency as a primary design goal, as well as adaptive and re-configurable interfaces and multi-protocol capabilities.

How this heterogeneous and huge network formed by billions of devices and users will communicate is also an important issue that has to be faced from the beginning. Developments oriented to enable interoperability among devices and users will be a critical pillar in the SIoT design. Again, the energy efficiency plays an essential role; operating systems, communication protocols and algorithms that cooperate in the overall energy saving will be determinant in the establishment of SIoT. Lightweight and open middleware platforms as well as self-adaptive software will also favor the expansion of this technology.
Next, we analyze some of the most relevant research and development challenges that will help to mature this technology and lead the way to the future massive deployment and everyday use of the SIoT ecosystem.

\section{Open Research Issues}

With the aim of making real the SIoT paradigm, there are still numerous challenges that must be faced prior to the worldwide deployment of this technology.

1) Interoperability, Data Management, and Signal Processing: Due to the heterogeneous nature of the IoT devices, including different information processing and communication capabilities, together with user features and data, relationships and competences coming from the SNs, the system must be able to manage this variety of data types, providing interoperability among all the components. In order to facilitate communication and cooperation, common practices and standards are required [51]. This challenge is aligned with the identification requirement presented in Section V-B, since all components in the SIoT system need to be identified prior to interoperate with each other.

After all components in the network can interoperate, data management arises as another remarkable issue; how all the data coming from devices and users are going to be handled? Here, we can identify at least two issues: data storage and data management. Regarding to storage, it is obvious that it is practically impossible to store all the SIoT data in a unique server, so distributed approaches should be proposed in order to get an efficient storage system. Respecting data management, metadata structures such as those proposed by Metadata Standards ${ }^{3}$ can be the basis of data structures definition. Afterwards, standards proposed by the $\mathrm{W} 3 \mathrm{C}^{4}$ such as RDF (Resource Description Framework), DAML (Darpa Agent Markup Language, or OWL (Ontology Working Language) will help providing with meaning to the data coming from users and devices.

Of course, advanced data analysis (such as those proposed for Big Data [52]) and intelligent approaches will provide utility to the SIoT, so serving users and devices with meaningful and valuable information.

2) Discovery and Search Engines: Considering the huge volume of information in the SIoT, available data, services and applications need to be easily reachable. The use of searching and discovery mechanisms is mandatory when working with such a data size. Existing discovery approaches for web services such as UDDI, DPWS or RESTful-based [53], [54], can be adapted to deal with the requirements of SIoT related to data, services and applications lookup and discovery.

3) Energy Management: Devices taking part in the SIoT are typically moving around, and not connected to an unlimited power supply. So do users, carrying hand-held devices, that usually operate with batteries. Hence, the energy conservation is a conditioning factor in the design and operation of SIoT, and efficient energy management should be implemented at all levels; from M2M device communications to interface design.

\footnotetext{
${ }^{3} \mathrm{http}: / /$ metadata-standards.org/

${ }^{4}$ http://www.w3.org/
} 
All stages in the design of SIoT technologies have to be oriented to low-energy consumption. While energy harvesting technologies do not yet provide enough resources, this is a broad research topic where some approaches proposed for WSNs and other low-power technologies can be adapted to deal with the requirements of the SIoT (e.g., scalability, availability, and heterogeneity).

4) Security, Privacy and Trust: These might be the most sensitive requirements for the success of SIoT; without a secure technology that ensures user privacy, safe communications and trustworthy interactions, the SIoT paradigm will not reach enough popularity to be considered a well-established technology, and all its potential will be lost.

Methods to guarantee data confidentiality and user privacy that are also required for other platforms can likely be reused in the SIoT context, always considering the special requisites of this paradigm. Lightweight mechanisms for data confidentiality and integrity, as well as effective ID management and privacy enhancing technologies represent the basis that will make users trust the SIoT ecosystem.

5) Self-operation, management and organization: As it has been commented before, the SIoT expects to be a worldwide technology composed of billions of devices and people. When imaging how the global management of such a huge platform should be, one easily realizes that automatic operation is needed at most levels.

Mechanisms including self-organization, self-management, self-operation, self-healing, and self-protection capabilities will definitely be a decisive part of the SIoT. But not only automatic network management will be relevant, also autonomic data analysis, and service discovery and composition will contribute to enhance the user experience. Again, the adaptation of approaches proposed for other technologies can be an starting point to automatize the operation of the SIoT ecosystem.

6) Heterogeneity: Sensors, actuators, ID-tags, smartphones, tablets, computers, etc., will be part of the SIoT, different brands and technologies will have to work together to achieve a common goal: provide users with advanced services and applications. For this, SIoT developments have to be able to integrate many types of devices, technologies, and services [5]. Interoperability at device level ensures that different technologies are able to communicate among them; the system should ideally be open to support a huge variety of different applications, whose characteristics and requirements may be extremely diverse, in terms of bandwidth, latency, reliability, availability, etc.

The necessity of dealing with heterogeneous devices will probably degrade the overall system performance compared to a highly optimized vertical design, but the extended functionality offered by the SIoT ecosystem will compensate this drawback. New designs that efficiently deal with heterogeneous technologies will be of great importance for the successful deployment of SIoT.

7) Interactions and Interfaces: The SIoT infrastructure will be focused on providing users with an advanced experience able to consume and produce data and services coming from devices and other users. Thus, the human-centric interface should provide a user-friendly medium to interact with devices and users.

How users and devices interact with each other is still an open challenge. Some approaches such as [55] and [26] propose a set of possible interactions among the different elements, but most of them are focused on specific applications. A global set of interactions needs to be defined, as well as methods to manage these interactions, for example, users can get data from their own devices, but how to get data from other users' devices is not completely clear, and it is very aligned with the privacy issues commented before; should I give completely free access to my devices? What about sensitive data such as my current location? Can I provide/get anonymized data?

8) Service Management (discovery and composition): Nowadays there exist a number of approaches to discover and perform service composition in the framework of the IoT [53]. The SIoT demands new capabilities in this area, and intelligent approaches that provide advanced functionality seem to be the best-performance option. SOA-based systems and DPWS [56] are a good start for service management in the SIoT, together with semantic-compliant approaches that are able to deal with context and data meaning.

9) Application Development: All the functionality offered by SIoT is meaningless without applications that make use of them. The application development process will vary depending on each situation; what devices and services are involved and which set of users the application is oriented to are determinant in this process. The use of open APIs will be helpful, and the implication of the users will bring new use cases that contribute to make the SIoT more accessible and functional.

10) New Business Models and Stakeholders: How to get benefits from the SIoT technology is a decisive point in the establishment of this new paradigm. When designing an advantageous platform, where both developers, stakeholders and users feel comfortable, and bearing in mind the collaborative nature of the SIoT $^{5}$, it is essential to take into account several considerations: (i) offer attractive and useful services and applications that encourage people to use them, (ii) look for non-conflicting business models that boost collaboration, (iii) recognize the customer experience by inviting the customers to participate, and (iv) target the adequate customer segment.

Once users find attractive the use of SIoT-based applications, capitalizing plans can be studied and launched, including: marketing and sales, research and development, advertising, application fees, device commercialization, etc. [57].

11) Fault Tolerance: In such a dynamic and mobile world, with context changing rapidly and with the heterogeneity that characterizes the SIoT, the reliable operation of the different components should be guaranteed [51]. Efficient adaptation to challenging situations will make the difference for a trusty platform, as well as a correct architectural organization that supports redundancy at several levels to ensure that it offers reliable information to the final users.

\footnotetext{
${ }^{5} \mathrm{http} / / /$ www.caba.org/resources/Documents/IS-2009-93.pdf
} 
12) Semantics and Context Management: The SIoT is aimed at providing functionality in a number of situations, and a set of devices might be used for several purposes at the same time. Thus, the ability to correctly manage the current context will definitely not only improve the performance of the system, but it will also make it more usable, by providing unambiguously access and data interpretation. Semantic approaches oriented to RDF and OWL [58] can be extended to include descriptors for SIoT users and devices characteristics, so facilitating the interoperability among all the components.

\section{CONCLUSIONS}

The Internet of Things paradigm aims at connecting anything, to be accessed at any time from anywhere. It is based on making available a set of services coming from the interoperation of billions of devices, with the ultimate objective of improving users everyday lives.

In this article, we review the juncture of Internet of Things with Social Networks that defines the paradigm, known as Social Internet of Things. This union emerges from inheriting social networking features and values of interactivity, recommendation \& filtering and services composition and suggesting a universal framework to combine users, devices and services and the interactions among them. This seamless integration can bring new relationships allowing the creation of novel services and applications that will definitely be of great interest both for final users and stakeholders.

Illustrating the evolution from Wireless Sensor Networks to Social Internet of Things, and providing a detailed description of this new paradigm from several points of view constitutes the first part of the article, to later going through the current situation of the state of the art in this technology, analyzing different proposals and implementations, to finally come up with a general architecture description and a deep review of the challenges and open research issues that must be solved to make real the Social Internet of Things.

\section{REFERENCES}

[1] I. T. Union, "ITU internet report 2005: The internet of things," 2005.

[2] J. Gubbi, R. Buyya, S. Marusic, and M. Palaniswami, "Internet of Things (IoT): A vision, architectural elements, and future directions," Future Generation Computer Systems, 2013.

[3] O. Vermesan, P. Friess, P. Guillemin, S. Gusmeroli et al., "Internet of things strategic research roadmap," O. Vermesan, P. Friess, P. Guillemin, S. Gusmeroli, H. Sundmaeker, A. Bassi, et al., Internet of Things: Global Technological and Societal Trends, pp. 9-52, 2011.

[4] J. Zheng, D. Simplot-Ryl, C. Bisdikian, and H. Mouftah, "The internet of things [guest editorial]," Communications Magazine, IEEE, vol. 49, no. 11 , pp. $30-31,2011$.

[5] M. Zorzi, A. Gluhak, S. Lange, and A. Bassi, "From today's intranet of things to a future internet of things: a wireless-and mobility-related view," Wireless Communications, IEEE, vol. 17, no. 6, pp. 44-51, 2010.

[6] D. Uckelmann, M. Harrison, and F. Michahelles, "An architectural approach towards the future internet of things," in Architecting the Internet of Things. Springer, 2011, pp. 1-24.

[7] S. Hong, D. Kim, M. Ha, S. Bae et al., "Snail: an ip-based wireless sensor network approach to the internet of things," Wireless Communications, IEEE, vol. 17 , no. 6, pp. 34-42, 2010.

[8] G. Wu, S. Talwar, K. Johnsson, N. Himayat, and K. D. Johnson, "M2M: From mobile to embedded internet," Communications Magazine, IEEE, vol. 49, no. 4, pp. 36-43, 2011.

[9] M. Weiser, "The computer for the 21st century," Scientific american, vol. 265, no. 3, pp. 94-104, 1991.
[10] J. Conti, "The internet of things," Communications Engineer, vol. 4, no. 6 , pp. 20-25, 2006

[11] K. Ducatel, M. Bogdanowicz, F. Scapolo, J. Leijten, and J.-C. Burgelman, Scenarios for Ambient Intelligence in 2010. Office for official publications of the European Communities, 2001.

[12] L. Yan, Y. Zhang, L. T. Yang, and H. Ning, The Internet of Things: from RFID to the next-generation pervasive networked systems. CRC Press, 2008.

[13] J. Yick, B. Mukherjee, and D. Ghosal, "Wireless sensor network survey," Computer networks, vol. 52, no. 12, pp. 2292-2330, 2008.

[14] I. F. Akyildiz, W. Su, Y. Sankarasubramaniam, and E. Cayirci, "Wireless sensor networks: a survey," Computer networks, vol. 38, no. 4, pp. 393 422, 2002.

[15] B. Latré, B. Braem, I. Moerman, C. Blondia, and P. Demeester, "A survey on wireless body area networks," Wireless Networks, vol. 17, no. 1, pp. 1-18, 2011.

[16] H. Hartenstein and K. P. Laberteaux, "A tutorial survey on vehicular ad hoc networks," Communications Magazine, IEEE, vol. 46, no. 6, pp. 164-171, 2008.

[17] C. Gomez and J. Paradells, "Wireless home automation networks: A survey of architectures and technologies," Communications Magazine, IEEE, vol. 48, no. 6, pp. 92-101, 2010.

[18] H. Schaffers, N. Komninos, M. Pallot, B. Trousse et al., "Smart cities and the future internet: towards cooperation frameworks for open innovation," in The future internet. Springer, 2011, pp. 431-446.

[19] A. Bassi and G. Horn, "Internet of things in 2020: A roadmap for the future," European Commission: Information Society and Media, 2008.

[20] D. Bandyopadhyay and J. Sen, "Internet of things: Applications and challenges in technology and standardization," Wireless Personal Communications, vol. 58, no. 1, pp. 49-69, 2011.

[21] D. Miorandi, S. Sicari, F. De Pellegrini, and I. Chlamtac, "Internet of things: Vision, applications and research challenges," Ad Hoc Networks, vol. 10, no. 7, pp. 1497-1516, 2012.

[22] M. Kranz, L. Roalter, and F. Michahelles, "Things that twitter: social networks and the internet of things," in What can the Internet of Things do for the Citizen (CIoT) Workshop at The Eighth International Conference on Pervasive Computing (Pervasive 2010), 2010.

[23] M. Baqer and A. Kamal, "S-sensors: Integrating physical world inputs with social networks using wireless sensor networks," in Intelligent Sensors, Sensor Networks and Information Processing (ISSNIP), 2009 5th International Conference on. IEEE, 2009, pp. 213-218.

[24] H. Ning and Z. Wang, "Future internet of things architecture: like mankind neural system or social organization framework?" Communications Letters, IEEE, vol. 15, no. 4, pp. 461-463, 2011.

[25] M. Baqer, "Enabling collaboration and coordination of wireless sensor networks via social networks," in Distributed Computing in Sensor Systems Workshops (DCOSSW), 2010 6th IEEE International Conference on. IEEE, 2010, pp. 1-2.

[26] L. Atzori, A. Iera, and G. Morabito, "Siot: Giving a social structure to the internet of things," Communications Letters, IEEE, vol. 15, no. 11, pp. 1193-1195, 2011

[27] L. Atzori, A. Iera, G. Morabito, and M. Nitti, "The social internet of things (siot)-when social networks meet the internet of things: Concept, architecture and network characterization," Computer Networks, 2012.

[28] L. Atzori, D. Carboni, and A. Iera, "Smart things in the social loop: Paradigms, technologies, and potentials," Ad Hoc Networks, 2013.

[29] D. Guinard, "A web of things application architecture: integrating the real-world into the web," Ph.D. dissertation, 2011.

[30] A. Pintus, D. Carboni, and A. Piras, "Paraimpu: a platform for a social web of things," in Proceedings of the 21st international conference companion on World Wide Web. ACM, 2012, pp. 401-404.

[31] C. Zhang, C. Cheng, and Y. Ji, "Architecture design for social web of things," in Proceedings of the 1st International Workshop on Context Discovery and Data Mining. ACM, 2012, p. 3.

[32] L. Console et al., "Interacting with social networks of intelligent things and people in the work of gastronomy," in Transactions on Interactive Intelligent Systems (TiiS). ACM, vol. 3, num. 1, pp. 1-38, 2012.

[33] J. I. Vazquez and D. Lopez-De-Ipina, "Social devices: autonomous artifacts that communicate on the internet," in The Internet of Things. Springer, 2008, pp. 308-324.

[34] D. Guinard, M. Fischer, and V. Trifa, "Sharing using social networks in a composable web of things," in Pervasive Computing and Communications Workshops (PERCOM Workshops), 2010 8th IEEE International Conference on. IEEE, 2010, pp. 702-707.

[35] L. Atzori, A. Iera, and G. Morabito, "Making things socialize in the Internet - does it help our lives?" in Kaleidoscope 2011: The Fully 
Networked Human?-Innovations for Future Networks and Services ( $K$ 2011), Proceedings of ITU. IEEE, 2011, pp. 1-8.

[36] A. Ciortea, O. Boissier, A. Zimmermann, and A. M. Florea, "Reconsidering the social web of things: position paper," in Proceedings of the 2013 ACM conference on Pervasive and ubiquitous computing adjunct publication, ser. UbiComp '13 Adjunct. ACM, 2013, pp. 15351544. [Online]. Available: http://doi.acm.org/10.1145/2494091.2497587

[37] S. Misra, R. Barthwal, and M. S. Obaidat, "Community detection in an integrated internet of things and social network architecture," in GLOBECOM.IEEE, 2012, pp. 1647-1652.

[38] M. Blackstock, R. Lea, and A. Friday, "Uniting online social networks with places and things," in Proceedings of the Second International Workshop on Web of Things. ACM, 2011, p. 5.

[39] I. Lequerica, M. Garcia Longaron, and P. M. Ruiz, "Drive and share: efficient provisioning of social networks in vehicular scenarios," Communications Magazine, IEEE, vol. 48, no. 11, pp. 90-97, 2010.

[40] N. Mäkitalo, J. Pääkkö, M. Raatikainen, V. Myllärniemi, T. Aaltonen, T. Leppänen, T. Männistö, and T. Mikkonen, "Social devices: collaborative co-located interactions in a mobile cloud," in Proc. of the 11th Int. Conf. on Mobile and Ubiquitous Multimedia. ACM, 2012, p. 10.

[41] J. An, X. Gui, W. Zhang, J. Jiang, and J. Yang, "Research on social relations cognitive model of mobile nodes in internet of things," Journal of Network and Computer Applications, vol. 36, no. 2, pp. 799-810, 2013.

[42] A. Jian, G. Xiaolin, Z. Wendong, and J. JinHua, "Nodes social relations cognition for mobility-aware in the internet of things," in Internet of Things (iThings/CPSCom), 2011 International Conference on and 4th International Conference on Cyber, Physical and Social Computing. IEEE, 2011, pp. 687-691.

[43] M. Nitti, R. Girau, L. Atzori, A. Iera, and G. Morabito, "A subjective model for trustworthiness evaluation in the social internet of things," in Personal Indoor and Mobile Radio Communications (PIMRC), 2012 IEEE 23rd International Symposium on. IEEE, 2012, pp. 18-23.

[44] F. Bao, I.-R. Chen, and J. Guo, "Scalable, adaptive and survivable trust management for community of interest based internet of things systems," in Autonomous Decentralized Systems (ISADS), 2013 IEEE Eleventh International Symposium on. IEEE, 2013, pp. 1-7.

[45] L. Console, F. Antonelli, G. Biamino, F. Carmagnola, F. Cena, E. Chiabrando, V. Cuciti, M. Demichelis, F. Fassio, F. Franceschi et al., "Interacting with social networks of intelligent things and people in the world of gastronomy," ACM Transactions on Interactive Intelligent Systems (TiiS), vol. 3, no. 1, p. 4, 2013.

[46] U. Ceipidor, C. Medaglia, V. Volpi, A. Moroni, S. Sposato, and M. Tamburrano, "Design and development of a social shopping experience in the iot domain: The shoplovers solution," in Software, Telecommunications and Computer Networks (SoftCOM), 2011 19th International Conference on. IEEE, 2011, pp. 1-5.

[47] V. Stavroulaki, D. Kelaidonis, V. Foteinos, G. Poulios, P. Demestichas, and K. Moessner, "Enabling smart cities through a cognitive management framework for the internet of things," IEEE Communications Magazine, p. 103, 2013.

[48] D. Hussein, S. N. Han, X. Han, G. M. Lee, and N. Crespi, "A framework for social device networking," in Distributed Computing in Sensor Systems (DCOSS), 2013 IEEE International Conference on. IEEE, 2013, pp. 356-360.

[49] P. Semmelhack, Social Machines: How to Develop Connected Products That Change Customers' Lives. Wiley, 2013.

[50] G. M. Lee, W. S. Rhee, N. Crespi, Proposal of a new work item on social and device networking, Q11-13-May13-C-08, ITU-T, 2013.

[51] F. Mattern and C. Floerkemeier, "From the internet of computers to the internet of things," in From active data management to event-based systems and more. Springer, 2010, pp. 242-259.

[52] P. Russom, "Big data analytics," TDWI Best Practices Report, Fourth Quarter, 2011.

[53] D. Guinard, V. Trifa, S. Karnouskos, P. Spiess, and D. Savio, "Interacting with the soa-based internet of things: Discovery, query, selection, and on-demand provisioning of web services," Services Computing, IEEE Transactions on, vol. 3, no. 3, pp. 223-235, 2010.

[54] S. Evdokimov, B. Fabian, S. Kunz, and N. Schoenemann, "Comparison of discovery service architectures for the internet of things," in Sensor Networks, Ubiquitous, and Trustworthy Computing (SUTC), 2010 IEEE International Conference on. IEEE, 2010, pp. 237-244.

[55] M. Kranz, P. Holleis, and A. Schmidt, "Embedded interaction: Interacting with the internet of things," Internet Computing, IEEE, vol. 14, no. 2, pp. 46-53, 2010.

[56] G. Cândido, F. Jammes, J. B. de Oliveira, and A. W. Colombo, "Soa at device level in the industrial domain: Assessment of opc ua and dpws specifications," in Industrial Informatics (INDIN), 2010 8th IEEE International Conference on. IEEE, 2010, pp. 598-603.

[57] J. Heidemann, M. Klier, and F. Probst, "Online social networks: A survey of a global phenomenon," Computer Networks, 2012.

[58] A. Katasonov, O. Kaykova, O. Khriyenko, S. Nikitin, and V. Y. Terziyan, "Smart semantic middleware for the internet of things." ICINCO-ICSO, vol. 8, pp. 169-178, 2008.

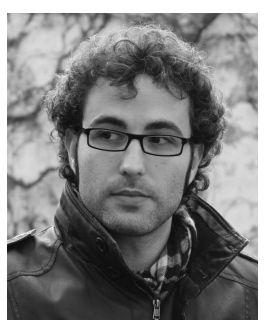

Antonio M. Ortiz received his $\mathrm{MsC}$ degree in Advanced Computer Technologies in 2008 and his $\mathrm{PhD}$ degree in Computer Science in 2011, both from University of Castilla-La Mancha, Albacete, Spain. During 2012, he was post-doctoral research fellow at the LYIT, Ireland. Since the end of 2012, he is a post-doctoral researcher at the RS2M department in the Institut Mines-Telecom, Telecom SudParis, France. His research interests are focused on Internet of Things, Social Networks, Wireless Sensor Networks, Cyber Physical Systems, and Artificial Intelligence. $\mathrm{He}$ is also involved in a number of European projects and contributes to the ITU-T and oneM2M standardization bodies.

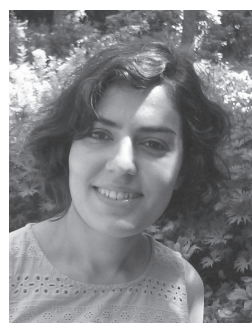

Dina Hussein received M.S. degree in Information Systems, specializing in online social networks design patterns, from the Arab Academy for Science, technology and Maritime Transport (AASTMT) Cairo, Egypt, in March 2011. She is currently pursuing her Ph.D at the Department of Wireless Networks and Multimedia Services of the Institut Mines-Telecom, Telecom Sud-Paris. Her research focuses on social cognition, context awareness in Ubiquitous Computing and building adaptive Social Internet of Things based services for enhancing QoE.

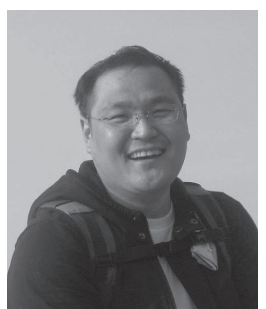

Soochang Park received the Ph.D. degree in Computer Science and Engineering from Chungnam National University in Aug. 2011. He is currently a research associate in Institut Mines-Telecom, Telecom SudParis. His research interests are the areas of computer communication and networking. $\mathrm{He}$ is mainly interested in IP routing with AS-level configuration; mobility management in both IPv4 and IPv6 including host-based and network-based; and routing, mobility management, and QoS in MANETs and WSNs.

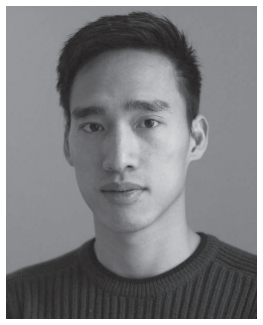

Son N. Han received M.S. degree in Computer Science from The University of Seoul in 2009 and Dip.-Ing. degree in Applied Mathematics from Hanoi University of Technology in 2006. He is currently pursuing his Ph.D. at the Department of Wireless Networks and Multimedia Services of Telecom SudParis, Institut Mines-Telecom. His research interests include Embedded Web Services, Service Composition, Semantic Web, and the application of Web technology for Internet of Things. Previously, he worked as a researcher for Korea Electronics and Telecommunications Research Institute (ETRI) from 2009 to 2011.

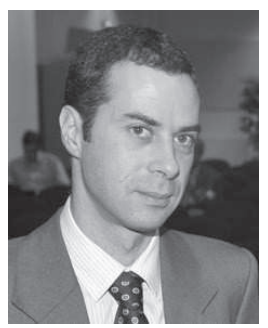

Noel Crespi Senior Member, IEEE, holds a Master's from the Universities of Orsay and Kent, a diplôme d'ingénieur from Telecom ParisTech, and a Ph.D. and a Habilitation from Paris VI University. He worked from 1993 in CLIP, Bouygues Telecom, France Telecom R\&D in 1995, and Nortel Networks in 1999. He joined Institut Mines-Telecom in 2002 and is currently professor and program director, leading the Service Architecture Laboratory. He is appointed as coordinator for the standardization activities in ETSI and 3GPP. He is also a visiting professor at the Asian Institute of Technology and is on the four-person Scientific Advisory Board of FTW, Austria. His current research interests are in service architectures, P2P service overlays, future Internet, and WebNGN convergence. He is the author/coauthor of more than 250 papers and contributions in standardization. 